\title{
Active and Logistical Networking for Grid Computing: The E-toile Architecture
}

\author{
Alessandro Bassi ${ }^{1}$, Micah Beck ${ }^{3}$, Fabien Chanussot ${ }^{1}$, Jean-Patrick Gelas ${ }^{1}$, \\ Robert Harakaly ${ }^{2}$, Laurent Lefèvre ${ }^{1}$, Terry Moore ${ }^{3}$, James Plank ${ }^{3}$, and \\ Pascale Primet ${ }^{1}$ \\ 1 INRIA / LIP (UMR CRNS, INRIA, ENS, UCB Lyon1) \\ Ecole Normale Supérieure de Lyon - 46, allée d'Italie - 69364 LYON Cedex 07 - France \\ laurent.lefevre@inria.fr, \{fabien.chanussot, pascale.primet\}@ens-lyon.fr \\ 2 UREC CNRS
}

Ecole Normale Supérieure de Lyon - 46, allée d'Italie - 69364 LYON Cedex 07 - France robert.harakaly@urec.cnrs.fr

3 LoCI Laboratory - University of Tennessee

203 Claxton Building - 37996-3450 Knoxville, TN, USA

\{abassi,mbeck, tmoore, plank\}@cs.utk.edu

\begin{abstract}
While active networks provide new solutions for the deployment of dynamic services in the network by exposing network processing resources, logistical networks focus on exposing storage resources inside networks by optimizing the global scheduling of data transport, and data storage. In this paper, we show how active and logistical environments working together can improve Grid middleware and provide new and innovative high-level services for Grid applications. We validate and experiment this approach combining the Internet Backplane Protoco 2 suite with the Tamanoir Active Node environment. Our target architecture is the French e-Toile Grid infrastructure [1] based on high performance backbone (VTHD) [2].
\end{abstract}

\section{Introduction}

The emergence of Grid computing as the ultimate solution for the scientific computing community has driven attention and research in the recent past, and especially since the proliferation of high performance network capabilities. The common purpose is to aggregate geographically distant machines and to allow them to work together to solve large problems of any nature.

${ }^{1}$ This work is supported and carried out within the framework of the RNTL e-Toile project and the RNRT VTHD++ project. More information on e-Toile project are available on http://www.urec.cnrs.fr/etoile/

2 This work is supported by the National Science Foundation Next Generation Software Program under grant \# EIA-9975015, the Department of Energy Scientific Discovery through Advanced Computing Program under grant \# DE-FC02-01ER25465, and the NSF Internet Technologies Program under grant \# ANI-9980203. 
The optimization of the data transfers within a Grid, which we define as High Performance Grid Networking, is the focus of our interest in this area. As the load, the capacity and the availability of network links used during data transfers may heavily affect Grid application performances, a fully functional Grid is dependent on the nature and quality of the underlying network. Appropriate performance, together with a high degree of flexibility, are therefore key factors of a successful Grid project. One of the important characteristic of the data transfers within a Grid environment is that the spectrum of exchanged data volume can spread over 9 orders of magnitude, ranging from few bytes in interactive traffic up to terabytes scale bulk data transfer. Requirements in terms of reliability and delay can also be very heterogeneous.

To introduce our work in this area, and present our research efforts within the e-Toile [1] grid environment, we would like to step back and reason about some fundamental characteristic that current network services show. In particular, we would like to focus the attention on the encapsulation of current network services, while our approach allows low-level functionalities to be exposed to higher levels.

The architectural design of the e-Toile grid platform, based on a very high speed network and on active and logistical network technologies, allows the study of the existing communications limits regarding services and protocols and the validation of more efficient approaches aiming to carry gigabit performance to the grid user level and taking into consideration the specific needs of grid flows; these goals are achievable through services which are made possible by the exposure of certain equipments' functionalities. While in the context of the e-Toile project different innovative approaches are explored, such as network performance measurement, differentiated services evaluation, high performance and enhanced transport protocols, active networking technology and services and logistical networking, this paper will focus on the last two aspects.

The paper is organized as follows: in section 2 we will describe our driving philosophy about network services, while section 3 will focus on the concept of an active grid. in section 4 we will describe the e-Toile grid platform and active and logistical deployment on the e-Toile platform (section 5).

\section{Exposing Network Services}

To the extent that the scientific computing community is already using the network as a computer, the Internet provides a ubiquitous communication substrate connecting its components (with routers acting as special-purpose elements invisible in the architecture), while network servers provide all access to storage and computation. Illustrations of such servers and services are plentiful: FTP, NFS, and AFS provide access to storage; Condor, NetSolve, Ninf provide lightweight access to processing; HTTP provides access to both; GRAM provides access to heavyweight computing resources; LDAP provides access to directory services; and so on. What is notable about these instances, and is equally true of almost all the other cases we could add to the list, is that they represent relatively encapsulated network services, where with encapsulated network service we mean 
architectures implementing functionalities that do not closely model the underlying network resource, but have to be implemented by aggregating the resource and/or applying significant additional logic in its utilization.

The best effort delivery of datagrams at the IP level, on the other hand, can be taken as example of a relatively exposed network service. An exposed network service adds enough additional abstraction to the underlying network resource to allow it to be utilized at the next higher level, but does not aggregate it or add logic beyond what is necessary for the most common and indispensable functionality that uses it.

An important difference between the two approaches emerges when we need to extend the functionality of a given service. Encapsulated services tend to be implemented by heavyweight servers and have APIs designed at a high semantic level, interposing themselves between the client and low overhead, transparent access to the underlying resources As a result, it can be difficult, inefficient, or in some cases impossible to build new functionality on top of such APIs. Instead, encapsulated services tend to implement new functionality through plug in modules that extend the functionality of the server, introducing new code that has access to low level interfaces within the server. These plug-in modules are the server equivalent of microcode in CISC processors, raising a familiar set of questions about access control and security for the management of such code.

Extending the functionality of an exposed service makes different demands because exposed services have lighter weight servers and APIs designed at a simpler semantic level. Since these factors are conducive to low overhead and more transparent access to the underlying resources, it tends to be much easier and more efficient to build new functionality on top of exposed services. Exposed services promote the layering of higher-level functionality on top of their APIs, either in higher-level servers or in client code. This layering of services, which is analogous to the user-level scheduling of a RISC processor by a compiler, is perhaps most familiar in construction of a network services stack.

\subsection{Exposing Network Storage Resources: The Internet Backplane Protocol}

Despite the familiarity of the exposed approach for the network services stack, it may still not be obvious how to apply it to a resource such as storage. After all, almost every technology for the access and/or management of network storage one can think of (FTP, HTTP, NFS, AFS, HPSS, GASS, SRB, NAS, etc.) encapsulates the storage behind abstractions with relatively strong semantic properties. For that reason, our research in this area had to start by creating a protocol, the Internet Backplane Protocol (IBP) [3], that supports the management of remote storage resources while leaving them as exposed as possible. IBP is a network service that provides an abstraction of shared network storage. Each IBP server (called also depot) provides access to an underlying storage resource to any client that connects to the server. In order to enable sharing, the depot hides details such as disk addresses, and provides a very primitive 
capability-based mechanism to safeguard the integrity of data stored at the depot. IBP's low level, low overhead model of storage is designed to allow more complex structures, such as asynchronous networking primitives and file and database systems, to be built on top of the IBP API. This key aspect of the IBP storage model, the capacity of allocating space on a shared network resource, can be seen as doing a C-like malloc on an Internet resource, with some outstanding differences, such, for instance, time-limitation, to prevent Denial-of-Use attacks.

With IBP in place the question becomes how easy or difficult it is to layer storage services with strong semantic properties on top of the weak underlying storage resources provided by IBP depots. Our experience shows that the answer varies between different cases. In some cases (e.g. building a file abstraction) earlier models can be followed and the design is relatively straightforward; in other cases (e.g. point-to-multipoint communication) the inclination to take a more encapsulated approach remains strong, and the consequently the design choice is more difficult.

\subsection{Exposing Network Processing Resources: Tamanoir}

The growing interest in the Active Networking field might be seen as a natural consequence of the difficulties experienced when integrating into a shared network infrastructure the existing technologies with new ones. In "active" networking vision, network equipments can perform computations on user data in transit, therefore exposing their computation capabilities accessible by the end users by supplying programs called services, that modify the behavior of the network. These equipments are called active nodes $(A N)$ and show a greater flexibility towards the deployment of new functionalities, more adapted to the architecture, the users and the service providers' requirements. The price to pay to have this greater flexibility is, generally, an increased attention needed towards security and performance.

The Tamanoir 4 4 framework is an high performance active environment based on active edge routers, able to handle different applications and various data stream at the same time. The two main transport protocol, TCP and UDP, are supported by the Tamanoir Active Node (TAN) for carrying data. One of the characteristics of Tamanoir is the capacity of making use and exposing logistical storage for optimizing end-user services requests, especially in terms of performance. As explained in section 5, each Tamanoir node can take advantage not only of IBP depot located on the same node, but also with any depot participating in backbones such as the Logistical Backbone.

\section{Concepts of an Active Grid}

Our attention towards an active grid paradigm was driven by the complains of Grid application designers about standard network characteristics, such as reliable packet transport between nodes using the TCP/IP protocol suite, not being suited for typical Grid applications. The active grid architecture we envision is 
based on a virtual topology of active network nodes spread on programmable routers of the network. Active routers are deployed on network periphery, and allow data stream processing and storage, either in an explicit way (following a request by the application) or encapsulated one. Considering that the future of WAN backbones lies on all-optical equipment, we concentrate active operations on routers and nodes mapped at network periphery.

Active nodes are connected between each other and each AN manages communications for a small subset of Grid nodes. Grid data streams cross the active layer twice, before and after passing through the passive backbone.

\subsection{Active Network Benefits for Grid Applications}

The communications needs of Grid applications can be improved by the use of an Active Grid architecture, in the following areas: application deployment (needs of active reliable multicast), Grid management and support, wide-area parallel processing (needs of QoS and data conversion services....).

Most of services needed by Grid environments, such as high performance transport, dynamic topology adapting, QoS, on-the-fly data compression, data encryption, data multicast, data conversion, and errors management can be easily and efficiently deployed on demand with an Active Grid architecture.

\subsection{Active Grid Architecture}

To support most Grid applications, an Active Grid architecture have to deal with the two main Grid configurations, Meta Cluster Computing and Global Computing. In the first case, where the configuration shows an high level of coupling, an active node is mapped on network head of each cluster or parallel machine. This node manage all data streams coming or leaving a cluster. All active nodes are linked with other AN mapped at backbone periphery. An Active node delivers data streams to each node of a cluster and can aggregate output streams to others clusters of the Grid. In the second one, characterized by a loosely coupled configuration, an AN can be associated with each Grid node or can manage a set of aggregated Grid nodes. Hierarchies of active nodes can be deployed at each network heterogeneity point. Each AN manages all operations and data streams coming to Grid Nodes, such as subscribing operations of voluntary machines, results gathering, nodes synchronization or checkpointing. For both configurations, active nodes could manage the Grid environment by deploying dedicated services adapted to Grid requirements : management of nodes mobility, dynamic topology re-configuration, fault tolerance.

\section{The E-toile Grid Platform}

The e-Toile project, funded by the French Ministry of Research in the realm of the RNTL (Réseau National des Technologies Logicielles) [5] initiative, focuses on three complementary objectives: 
- to build an experimental high performance grid platform that scales to nation-wide needs and geographical distribution, providing an high performance network and software support for the ACI-GRID [6] initiative

- to develop original Grid services in order to go beyond the various limits of existing middleware and to exploit completely the services and capacities offered by a very high performance network. The e-Toile middleware integrates the most recent and relevant works of the French computer science laboratories (INRIA, CNRS) on the area of advanced communication services.

- to evaluate the deployment cost of chosen computing intensive and dataintensive applications and to measure the gain obtained thanks to the grid.

The partners of the project, providing various resources (clusters, storage space...) and federating the available tools in an integrated plate-form, are INRIA (Reso, Remap, Apache, Paris), CNRS (PRISM, IBCP), Communication \& Systems, SUN Labs Europe, EDF (Electricite De France, French Electricity Board) and CEA (Atomic Energy Center).

The e-Toile middleware relies upon existing middleware, in particular Globus and the Sun Grid Engine, and integrates independent building blocks developed in the context of ACI-GRID. The most innovative grid components are in the areas of grid services (in terms of resource allocation), performance measurement, security, communication paradigms, distributed storage and active networking.

The figure 1 represents the architecture of the testbed with the resources currently interconnected. The platform is composed by two distinct testbed:

\section{E-Toile Grid physical architecture}

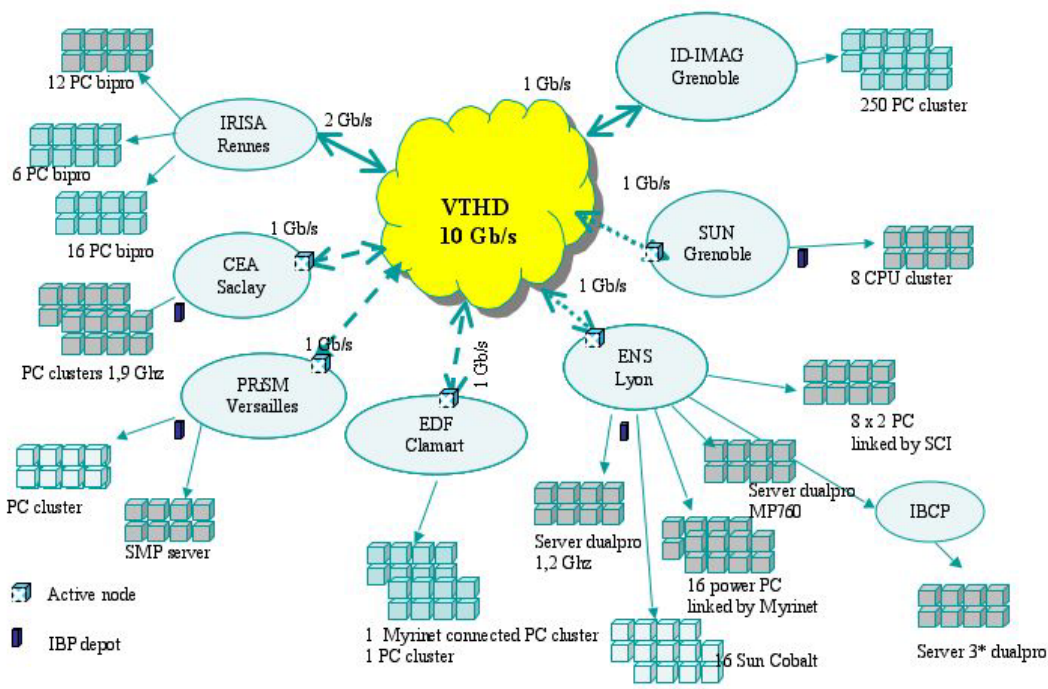

Fig. 1. Physical Architecture of the e-Toile grid 
a development testbed in order to allow testing of new middleware component and a production one for grid applications. The VTHD (Vraiment Trés Haut Débit) [2] network infrastructure interconnects the grid nodes with 1 or 2 Gbps links. This existing french high performance research backbone has been extended to all participant sites.

Network monitoring and performance measurement tools, developed by INRIA and CNRS within the European DataGRID project are deployed in e-Toile. The MapCenter [7] Grid status visualization tool permit users and managers to visualize the available resources in real time and services.

One of the original aim of the e-Toile project is to focus on the High Performance Grid Networking dimension and to evaluate the benefits grid middleware and grid application can extract from enhanced networking technology. The performance problem of the grid network can be studied from different point of view:

- Measuring and monitoring the end-to-end performances helps to characterize the links and the network behaviour. Network cost functions and forecasts, based on such measurement information, allow the upper abstraction level to build optimization and adaptation algorithms.

- Evaluating the advantages of differentiated services (Premium or Less than Assured Services) offered by the network infrastructure for specific streams.

- Creating enhanced and programmable transport protocols to optimize heterogeneous data transfers within the grid.

\section{Active and Logistical Resource Deployment in E-toile}

Logistical and active networking equipments are currently deployed in the e-Toile architecture. Each service willing to use logistical storage has to instantiate its own IBP client classes in order to communicate with an IBP depot. These classes provide constructors and methods to create capabilities on any IBP depot, with whom the Tamanoir service can write, read and manage data remotely on the IBP depot.

A first and very basic TAN service, called IBP_Service uses a IBP_store operation to redirect the beginning of a data stream towards the IBP depot. The IBP service checks as well the presence of the required service each time that a new packet arrive, and if so, a IBP_load operation is done to redirect all the data cached in the IBP depot towards the service able to process, route and forward efficiently these data. The only difference between the IBP_Service and any other service lies in the load-time, which is done at boot time for the IBP_Service, in order to be able to cache data immediately.

The use of IBP depot on each TAN should allow the storage of data to provide reliability through redundancy. If data are replicated on different server and one of them become either out of order or unreachable, data should still be downloadable from another server transparently.

Explicit routing and optimized scheduling are also possible through logistical active nodes. Several experiments made by the IBP team show that, in order to transmit as fast as possible huge amount of data, the path chosen by "classic" 
routers might show well below performance than the optimal one. Adding a staging point in the middle of two fast connections, knowing the topology of the underlying network, often improves performance dramatically. Unfortunately current transport protocols encapsulate the path, offering an end-to-end service that is not well adapted to the performance needs a grid application often has. A Tamanoir service, upon reception of a data stream, could store the stream on a number of different depots, participating in directories such as the Logistical Backbone (L-Bone), optimizing the total transfer time explicitly routing the data packets towards faster connections, and staging data in middle points at the boundaries of high-speed connections. We run several experiments mixing active and logistical equipment, and the latency measured [8] for packet caching of an active stream shows a very small overhead.In case of a change in the application scheduling, data stored at those intermediate nodes could be re-routed towards the new processing node in a much more efficient way.

\section{Conclusions and Future Works}

A grid, empowered with active and logistical networking, can not only improve significantly its global performances, but also and foremost provide new and innovative services to grid applications and middleware, giving an easy and direct control over high level services, such as reliable multicast and active QoS.

In addition to the technical challenges outlined in this article, the direction of our research is towards the integration of our active and logistical technologies with existing middleware, such as Globus and the Sun Grid Engine, giving them the ability to encapsulate active and logistical technologies for their internal needs, and to expose active and logistical services to upper layer's applications.

Our plan is also to integrate these technologies further, especially adding active and intelligent services to the logistical layer in fields such as efficient and reliable data deployment for the grid. IBP depots, through the Data Mover (DM) plug-in module, by sending a request to a Tamanoir Active Node could take advantage of any active services and could improve data transport.

\section{References}

1. RNTL e-Toile French Grid Project - http://www.urec.cnrs.fr/etoile.

2. Very high broadband RNRT VTHD project: (http://www.vthd.org)

3. Beck, M., Moore, T., Plank, J.: An end-to-end approach to globally scalable network storage. In: ACM SIGCOMM 20002 Conference, Pittsburgh, PA, USA. (2002)

4. Gelas, J.P., El Hadri, S., Lefèvre, L.: Towards the design of an high performance active node. Parallel Processing Letters 13 (2003)

5. RNTL: (http://www.industrie.gouv.fr/rntl)

6. ACI Grid initiative: (Initiative of french ministry of research for globalisation of computer resources and data - http://www-sop.inria.fr/aci/grid/public/)

7. MapCenter Grid visualization tool: (http://ccwp7.in2p3.fr/mapcenter/)

8. Bassi, A., Gelas, J.P., Lefèvre, L.: Tamanoir-IBP: Adding Storage to Active Networks. In: Active Middleware Services, Edinburgh, Scotland, IEEE computer society (2002) 27-34 ISBN: 0-7695-1721-8. 